\title{
Cellular Immunity and Immune Activation Markers in HIV Seropositive Tuberculosis Patients in Zaria, Nigeria
}

\author{
Tsavyange Peter Mbaave
}

\begin{abstract}
Background: The CD4+ T lymphocyte is used in disease staging in both HIV and Tuberculosis/HIV co infection. Its cost and technical requirements have necessitated search for alternative tests. The search for a cheaper surrogate for the CD4+ T lymphocytes count is imperative in resource limited settings dependent on donor funding as ours necessitated this study.
\end{abstract}

Materials and method: The study subjects were new patients who presented to Ahmadu Bello University Teaching Hospital (ABUTH) Zaria during the study period. They underwent clinical evaluation, relevant investigations. Those found to be co infected with TB/HIV further had CD4+ T lymphocytes count, beta-2-microglobulin (ß2M) and Erythrocyte Sedimentation Rates (ESR) evaluated and were consecutively included in the study. HIV negative tuberculosis patients and normal persons served as controls. Findings: Seventy five study subjects were included in the study. Their mean CD4+ count was $178 \pm 116.81$ cells/ul., serum beta $-\beta 2 \mathrm{M}$ level was $3.29 \mathrm{mg} / \mathrm{L}$, tuberculin skin test (TST) reaction was $5 \pm 6.7 \mathrm{~mm}$ and erythrocyte sedimentation rate (ESR) was $67.88 \pm 37.32 \mathrm{~mm} / \mathrm{hr}$, which were statistically significantly different from the TB only and controls.

Conclusion and recommendation: The ESR and the TST reaction had weak and moderate predictive values for CD4+ count respectively. The association is too weak for these to be recommended as surrogates for CD4+ $T$ lymphocytes count and progression in HIV/TB co infected patients.

Index Terms- Cellular immunity, immune markers,HIV, Tuberculosis .

\section{INTRODUCTION}

The Human Immunodeficiency Virus (HIV) and Mycobacterium tuberculosis are both intracellular organisms, which target the $\mathrm{CD} 4^{+}$cells of the monocytes, macrophage lineage and T lymphocytes. ${ }^{[1]}$ HIV targets, destroys and impairs the function of these cells thereby suppressing cell-mediated immunity, which is necessary for protection against intracellular organisms. ${ }^{[2]}$ Infected individuals are thus prone to infection with intracellular organisms including tuberculosis. ${ }^{[3],[4],[5]}$ Experimental studies have shown that exposure of macrophages and T-lymphocytes from HIV infected patients to mycobacterium in vitro increased viral replication.
Also pleural fluid from tuberculosis patients showed increased viral multiplication in activated lymphocytes. This is attributed to increased production of tumor necrosis factor alpha (TNF- $\alpha$ ), Interleukins 1 and 6 (IL-1 and IL-6) by macrophages activated by the tuberculosis bacilli. ${ }^{[6],[7]}$ [8], [9]

There is thus an established cycle of enhanced progression of each disease. HIV patients co-infected with tuberculosis deteriorate more rapidly with adverse outcomes than those without tuberculosis. ${ }^{[8]}$ This is indicated by a more rapid decline in cellular immunity $\left(\mathrm{CD}^{+} \mathrm{T}\right.$ cells) and an elevation of levels of markers of immune activation like neopterin, $\beta 2 \mathrm{M}, \mathrm{TNF} \alpha$, and $\mathrm{P}^{24}$ antigen than found in either disease alone. ${ }^{[10][11],[12],}$ These and other indicators of the inflammation and activated immune status such as the erythrocyte sedimentation rate (ESR),C-reactive protein (CRP) and tuberculin skin test (TST) reactivity have been evaluated for use as cheaper alternatives to CD4+count in assessing the stage of immune-suppression as well as for progression of disease without any consensus. [13],[ 14],, 15], [16]

The search for an easier test and cheaper alternative to CD4+ cell count for staging of and prognostication of HIV/ TB co infection is relevant in our country that depends mainly on donor agencies for financing of their HIV and TB programmes.

The study was undertaken to evaluate the cellular immune status and immune activation markers in newly diagnosed HIV seropositive tuberculosis patients in Zaria and identify a possible surrogate for CD4+ count for disease staging and monitoring of progression and treatment response.

\section{MATERIALS AND METHODS}

A cross sectional descriptive study was carried out at Ahmadu Bello University Teaching Hospital (ABUTH) Zaria, a tertiary health care institution in the north western part of Nigeria from March 2007 to December 2008. The hospital draws its clientele from mainly from Kaduna and the surrounding states. It was a hospital based study which utilized convenience sampling method whereby patients were enrolled as they came into the hospital and satisfied the inclusion criteria.

The study subjects were patients who presented to the Tuberculosis and HIV clinics of the ABUTH Zaria respectively, or were admitted to the medical wards of the 
hospital and on evaluation, found to be co-infected with HIV and Tuberculosis. They were treatment naïve for both infections or had not used antiretroviral drugs in the preceding six months.

Inclusion criteria for study subjects were: a. Age $\geq 15$ years,b. Symptoms; signs and laboratory evidence of tuberculosis, c. Double ELISA positivity to HIV-1 or 2 antibodies (or both), d. Provision of informed consent.

The Exclusion criteria were: a. Age < 15 years, b. Presence of non-HIV related conditions likely to suppress immunity like cancers, diabetes mellitus, overt renal disease, steroid therapy and pregnancy.

c. Use of antiretroviral drugs (ARVs) or immunosuppressive therapy 6 months prior to diagnosis of tuberculosis,

\section{d. Refusal to have HIV testing.}

Inclusion and exclusion criteria for the TB controls were the same except for HIV positivity.

Ethical Approval was obtained from the Ethics and Research committee of the Ahmadu Bello University Teaching Hospital Zaria. All the subjects signed or thumb printed an informed consent form.

\section{A. Study Protocol}

All participants were interviewed by the investigator using a drawn up protocol, which contained the biodata, major symptoms and HIV risk factors. A general and systemic physical examination was carried out and recorded. The participants underwent the following investigations: Full blood count, erythrocyte sedimentation rate (ESR) Chest X-ray, HIV ZN Sputum staining for Acid Fast bacilli $(\mathrm{AFB}) \times 3$ and mantoux testing. Those with diagnosis of HIV/TB co infection or TB alone were enrolled as study subjects and controls respectively and further had $\mathrm{CD} 4+$ cell count and $\beta 2 \mathrm{M}$ estimation.

HIV status was determined by Enzyme Linked Immunosorbent Assay (ELISA) test using the Determine HIV 1and 2 (Abbot Laboratories, Japan) and Immunocomb HIV 1 and 2 (Orgenics, Israel) test kits to double test for HIV antibodiesThe ESR was carried out using the standard Westegren method and recorded in millimeters per hour.Tuberculin skin testing (TST) was carried out using the Mantoux technique. The standard $0.1 \mathrm{ml}$ of $5 \mathrm{TU}$ purified protein derivative (PPD) was given by intradermal injection in the anterio-lateral part of the mid-forearm. The subjects were instructed not to scratch or use water on the injection site until reaction was read. The reaction, adjudged by the indurations across the forearm was read at 72 hours. All results were reported in millimeters. The CD4+ lymphocyte count was measured using the Partec CyFlow counter (Partec GmbH Munster, Germany) machine which uses the principle of flow cytometry. Quqntitative $\beta 2 \mathrm{M}$ levels were measured in 39 randomly selected study subjects, $20 \mathrm{~TB}$ and 20 healthy controls using the enzyme linked immunoassay (ELISA) technique. Demeditec Diagnostics GmbH kit (Kiel, Germany) was used. Serum for estimation was separated from whole blood and immediately stored at $-20^{\circ} \mathrm{C}$ until the pooled samples were analyzed, which was done according to manufacturer's instructions.All the investigations were carried out in the hospital's laboratories by staff who were experienced in carrying out such investigations using available standard procedures. Staff of the department of radiology and community health carried the chest radiographs and, tuberculin skin test (TST) using respectively Diagnosis of tuberculosis was based on the WHO recommendations for resource poor countries . ${ }^{[17]}$

\section{STATISTICAL ANALYSIS}

Data obtained comprising the Biodata, Laboratory results, was analyzed using the statistical Package for social sciences (SPSS) for windows software version 11.5. All values were expressed as means \pm standard deviation and qualitative data as percentages. Student's t-test and Chi-square were used to assess the difference between the various variables and groups as appropriate. Pearson's test of correlation was used to check association between variables and groups. One-way ANOVA test was used to compare multiple groups. Linear and multiple regressions were conducted to find out predictive values of relevant variables. A P-value of $\leq 0.05$ was taken as statistically significant and confidence intervals were reported at $95 \%$.

\section{IV.RESULTS}

Seventy five (75) co-infected participants certified the criteria and were enrolled as study subjects. . Their age range was $18-55$ years with a mean of $34.03 \pm 8.34$ years. The age groups 25 to 34 years $(45.3 \%)$ and 35 to 44 years (32\%) accounted for the bulk of this group (Figure1).There were $36(48 \%$ ) males and 39(52\%) females. Twenty-five $(69.4 \%)$ of males and 28 females $(71.8 \%)$ were married. (table1). Thirty-five (35) HIV negative tuberculosis subjects and twenty (20) HIV negative apparently healthy (normal) persons comprising of blood donors and other volunteers with no other risk factors for immune-suppression or evidence of tuberculosis were used as controls.

There was no statistically significant difference in the age and gender of the study subjects and the two control groups (table1).

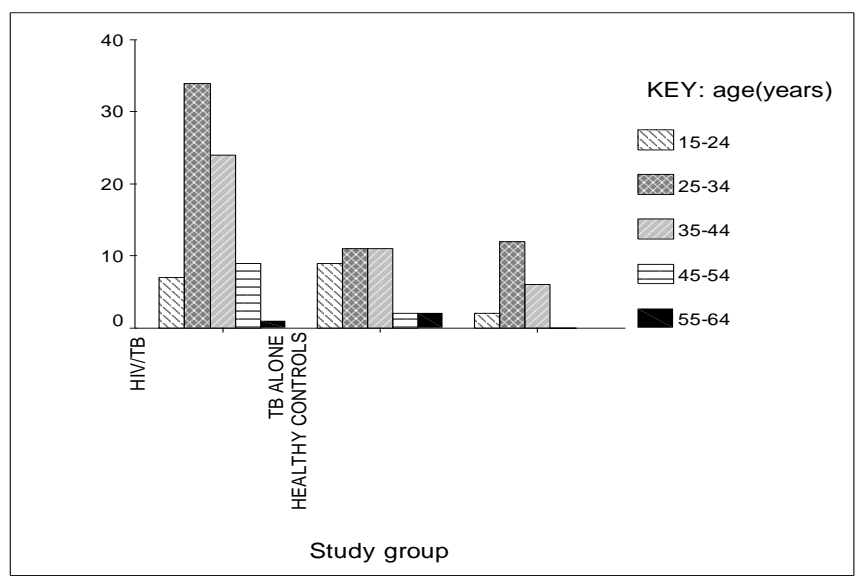

Figure 1: Age distribution of study subjects and controls 
Table 1: Marital Status of Study subjects and TB Controls.

\begin{tabular}{lrrrr}
\hline Marital & \multicolumn{2}{c}{ study subjects } & \multicolumn{2}{c}{ TB controls } \\
\cline { 2 - 5 } Status & Male & Female & Male & Female \\
& $\mathrm{N}(\%)$ & $\mathrm{N}(\%)$ & $\mathrm{N}(\%)$ & $\mathrm{N}(\%)$ \\
\hline Married & $25(69.4)$ & $28(71.8)$ & 12 & 12 \\
& & & $(63.2)$ & $(75.0)$ \\
Single & 10 & $10(25.6)$ & $7(36.8)$ & 4 \\
& $(27.7)$ & & & $(25.0)$ \\
Divorced & 1 & 1 & 0 & 0 \\
& $(2.8)$ & $(2.6)$ & $(0.0)$ & $(0.0)$ \\
Total & & 39 & 19 & 16 \\
& $(100)$ & $(100)$ & $(100)$ & $(100)$ \\
\hline
\end{tabular}

A.Immunological profile and immune activation markers. The mean CD4+count of the study subjects was $178.96 \pm 116.81$ cells $/ \mathrm{mm}^{3}$ with a range of $15-500$ cells $/ \mathrm{mm}^{3}$ as against $630 \pm 187.95$ (range 241-1049) in the TB controls $(\mathrm{p}=0.01) .61 .3 \%$ of study subjects had CD4+ count less than 200cells $/ \mathrm{mm}^{3}$ while $88 \%$ had $\mathrm{CD}+$ count less than 350 cells $/ \mathrm{mm}^{3}$. There was a statistically significant difference between the 3 groups when the study subjects and controls were matched according to their degree of immune status (tables 2). Using the ANOVA one way and post hoc tests, there was significant difference in mean levels of beta-2-microglobulins $(\beta 2 \mathrm{M})$ between the 3 groups. The study subjects had higher mean level than TB and healthy controls $(\mathrm{p}=0.01)$ (table 2) The mean erythrocyte sedimentation rate of the study subjects was $97.4 \pm 29 \mathrm{~mm} / \mathrm{hr}$ compared to $67.88 \pm$ $37.32 \mathrm{~mm} / \mathrm{hr}$ and $23.60 \pm 1.27 \mathrm{~mm} / \mathrm{hr}$ in the TB and healthy control groups respectively $(\mathrm{p}=0.01)$ (table 2).Using the post hoc test, there was also a statistically significant difference between the groups. The mean
TST reaction of study subjects was $5 \mathrm{~mm} \pm 6.7$ compared to $13 \mathrm{~mm} \pm 4.98$ and $11.73 \pm 0.42 \mathrm{~mm}$ in the TB and controls respectively $(\mathrm{p}=0.01)$. Using the post hoc test, there was a statistically significant difference between the study subjects and control groups $(\mathrm{p}=0.01)$, but none between the TB and the healthy control group $(\mathrm{p}=0.35)$ (table 2$)$.

\section{B. Correlation of $\mathrm{CD} 4+$ with immune activation markers:}

Forty-six $(61.3 \%)$ of the 75 study subjects had CD4+ cell count $<200 /$ ul with only $8(18 \%)$ having CD4+ count $>350$ cells/up (table2). Thirty-eight $(82 \%)$ of the study subjects had TST reaction of $<5 \mathrm{~mm}$ ( table 3 ). Pearson correlation was conducted to find out any association between CD4+ cell count, TST reaction, ESR and $\beta 2 \mathrm{M}$ of study subjects. A positive correlation was established between CD4+ cell count and TST reaction of the study subjects while a negative correlation was established (Pearson's $r=-0.30$, $\mathrm{p}=0.01$ ) between CD4+ and ESR of the study subjects (table 4). No such relationship was found in the TB controls. CD4+ count and $\beta 2 \mathrm{M}$ did not show any statistically significant relationship in both study subjects (Pearson's $\mathrm{r}=-0.028, \mathrm{p}=0.09$ ) and TB controls (table 4).Linear regression was used to establish the predictive value of TST reaction for $\mathrm{CD} 4+$ count $(\mathrm{r}=0.31, \mathrm{p}=0.01)$ and the equation for calculation of $\mathrm{y}$ (CD4+ count) from the known TST reaction size ( $\mathrm{x}$ ) of the study subjects (figure 2). It also established a weak predictive value of ESR for CD4+ $(\mathrm{r}=-0.08, \mathrm{p}=0.01)$, and the equation for calculation of $\mathrm{y}$ (CD4+ count) from the known ESR (x) of the subject. (figure3)The TST reaction best predicted advanced immunosuppression (CD4+ $<200$ cells/ul.).

Table 2: Mean CD4+, ESR, TST, $\mathrm{B}_{2} \mathrm{M}$ values of study subjects and controls.

\begin{tabular}{|c|c|c|c|c|}
\hline Parameter & study subjects & TB & Healthy controls & $\mathrm{P}$-value \\
\hline CD4+ (cells/ul) & $17896 \pm 118.81$ & $630.22 \pm 184.96$ & $662.70 \pm 161.44 *$ & $0.01 *$ \\
\hline $\operatorname{ESR}(\mathrm{mm} / \mathrm{hr})$ & $97.42 \pm 29.45$ & $67.86 \pm 37.32$ & $23.60 \pm 1.27 * *$ & $0.01 *$ \\
\hline TST reaction $(\mathrm{mm})$ & $5.04 \pm 6.71$ & $13.20 \pm 4.99$ & $11.73 \pm 0.42^{* *}$ & $0.01 *$ \\
\hline B2M(mg/L) & $3.29 \pm 0.40$ & $2.93 \pm 0.18$ & $2.55 \pm 0.20^{* *}$ & $0.01 *$ \\
\hline
\end{tabular}

Distribution of study subjects and controls by cellular immunity (CD4+) status

\begin{tabular}{lrrr}
\hline CD4+count & Study subjects & TB & Healthy controls \\
(Cells/up) & $\mathrm{N}(\%)$ & $\mathrm{N}(\%)$ & $\mathrm{N}(\%)$
\end{tabular}

\begin{tabular}{lccc}
$<200$ & $46(61.3)$ & $0(0.0)$ & $0(0.0)$ \\
$200-350$ & $21(28.0)$ & $1(2.9)$ & $0(0.0)$ \\
$351-500$ & $8(10.7)$ & $7(20.0)$ & $2(10.0)$ \\
$>500$ & $0(0.0)$ & $27(77.1)$ & $18(90.0)$ \\
Total & $75(100)$ & $35(100)$ & $20(100)$ \\
\hline
\end{tabular}


Cellular Immunity and Immune Activation Markers in HIV Seropositive Tuberculosis Patients in Zaria, Nigeria

Table 3: CD4+ counts and TST reaction of study subjects:

\begin{tabular}{lcccc} 
& \multicolumn{1}{c}{ Table 3: CD4+ counts and TST reaction of study subjects: } \\
\hline TST reaction & CD4+<200cells/up & CD4+200-350cells/up & \multicolumn{2}{c}{ CD4+351-500cells/up } \\
& $\mathrm{N}$ & $\mathrm{N}$ & $\mathrm{N}$ \\
\hline$<5 \mathrm{~mm}$ & 38 & 4 & 2 & 54 \\
$5-15 \mathrm{~mm}$ & 6 & 15 & 5 & 1 \\
$>15 \mathrm{~mm}$ & 2 & 2 & 5 \\
\hline Total & 46 & 21 & $\mathrm{~s} 8$ \\
\hline
\end{tabular}

Key: $\mathrm{N}=$ number of subjects

Table 4: Pearson's correlation of CD4+ count and immune activation markers in study subjects

\begin{tabular}{lcc}
\hline CD4+ VS Immune marker & Pearson's r & P value \\
& & \\
\hline CD4+VS B2M & -0.028 & 0.87 \\
CD4+VS ESR & -0.300 & $0.01 *$ \\
CD4+VS TST reaction & 0.560 & $0.01 *$ \\
& & 75 \\
$*$ = Statistically significant correlation, $\mathrm{N}=$ Number analysed & & 75
\end{tabular}

Key: $*$ = Statistically significant correlation, $\mathrm{N}=$ Number analysed

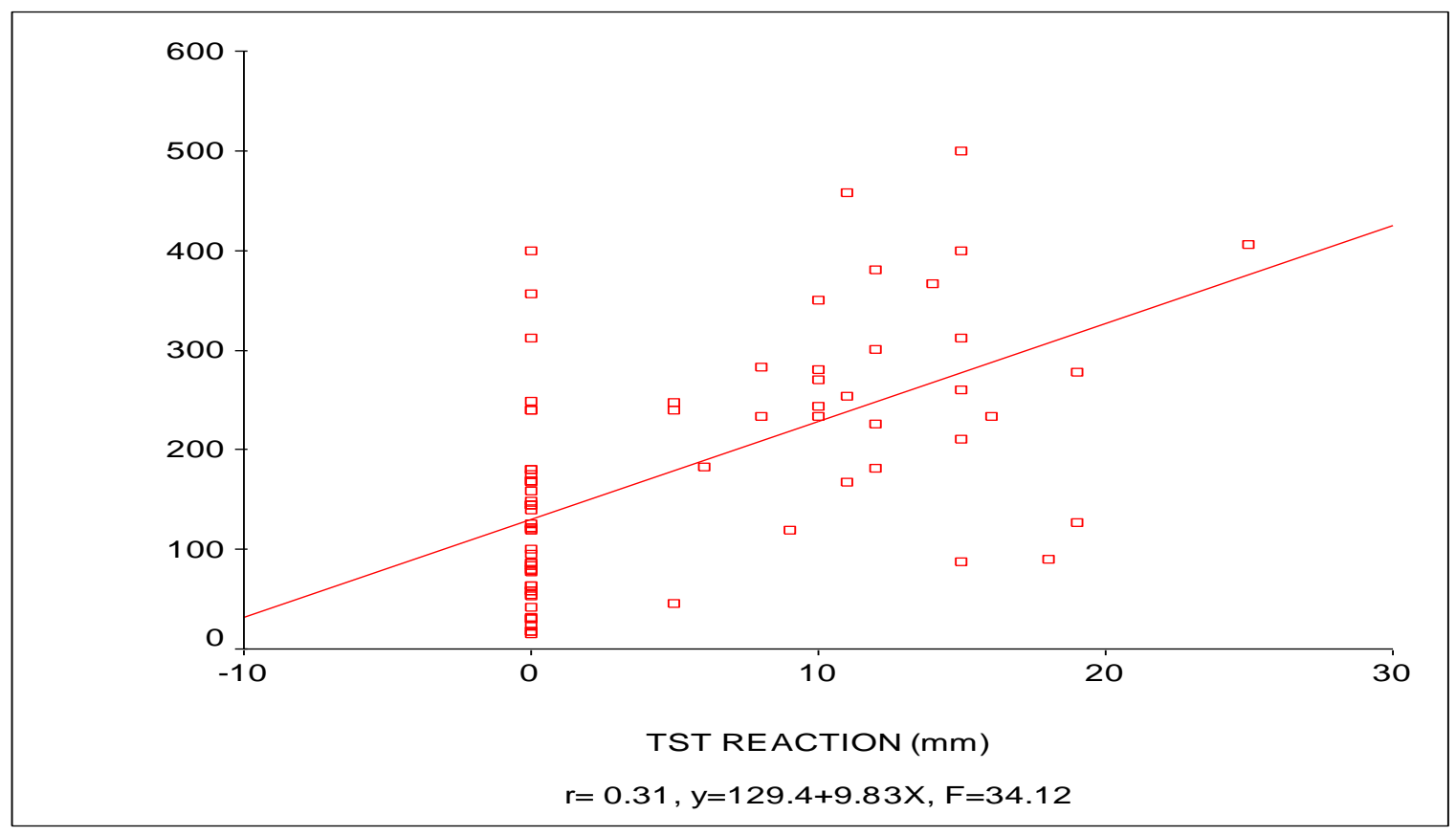

Figure 2: Regression of CD4+Cell Count on TST Reaction of Study subjects. 


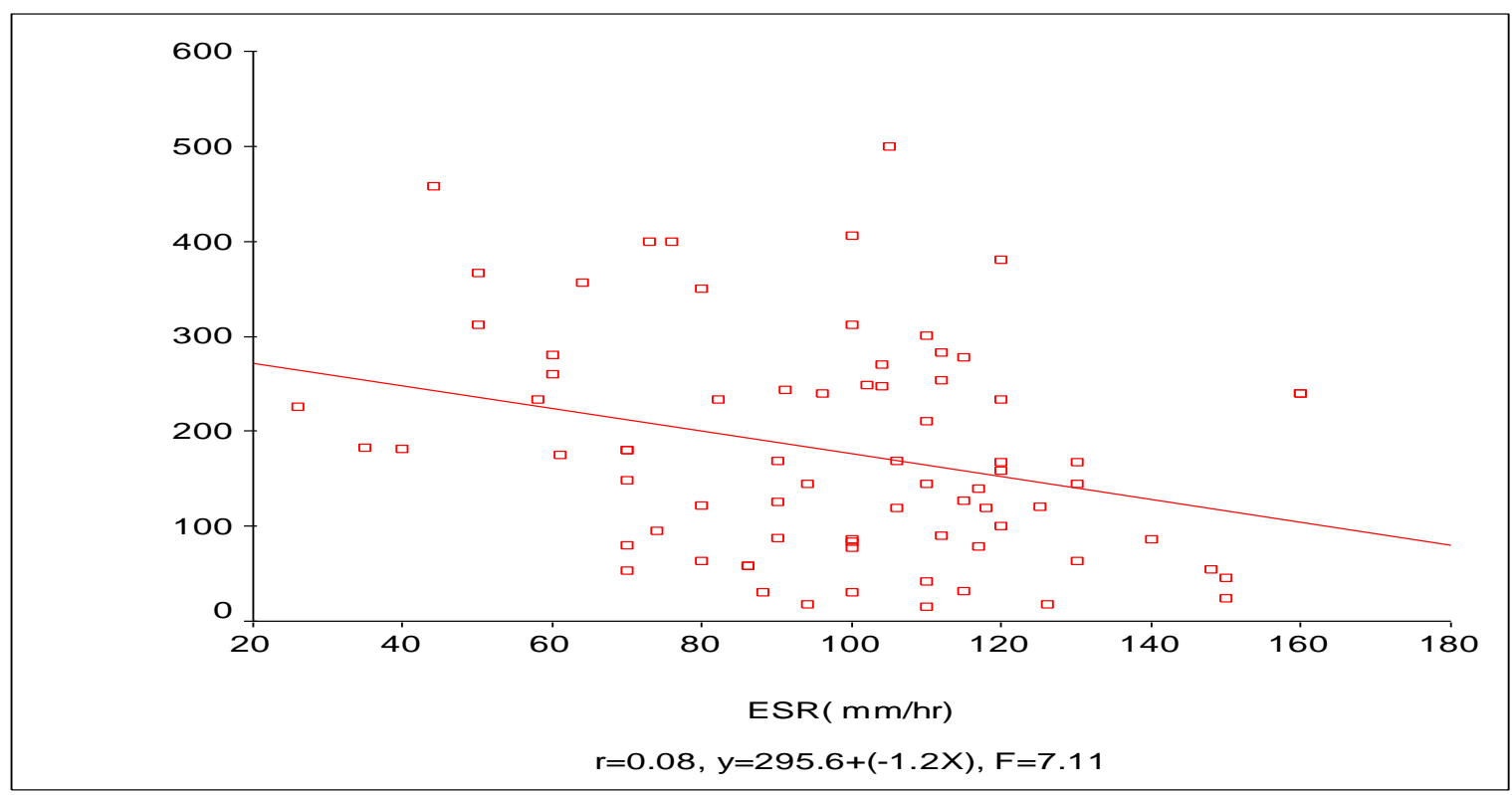

Figure 3: Regression of CD4+ count on ESR of study subjects

\section{DISCUSSION}

The mean CD4+ $\mathrm{T}$ lymphocytes count was significantly lower in the study subjects compared to the controls, with over $61 \%$ having CD4+ count of $<200$ cells/ul( $\mathrm{p}=0.01)$. Keiper et al in Philadelphia (USA) also reported 26 of 35 (74)\% of patients had CD4+ count less than 200cells /uL, ${ }^{[18]}$ while Teck in Malawi found that $89 \%$ of 158 HIV positive subjects with active or past TB without another HIV related disease had CD4+ count less than 350 cells/Ul. ${ }^{[19]}$ Although tuberculosis can occur at any level in the spectrum of HIV/AIDS immunity status, advanced immune suppression poses an increased risk for tuberculosis either as a progression to disease of a new TB infection or through reactivation of a quiescent TB focus. ${ }^{[20],[21]}$

The mean beta-2-microglobulin $(\beta 2 \mathrm{M})$ levels were noted to be higher in study subjects than TB and healthy controls but had no relationship with any of the parameters against which it was tested including the CD4+count. This agrees with findings by Wanchi et al that beta-2-microglobulin levels were more elevated in HIV/TB co-infection than when either disease occurred alone. ${ }^{[1]}$ Ritcher et al also demonstrated that HIV/TB co-infection was a strong factor for elevated beta -2 -microglobulins. ${ }^{[2]} \quad \beta 2 \mathrm{M}$ levels rise in response to cell activation or destruction, hence the marked elevation in co infection.. The levels in normal subjects in this study were

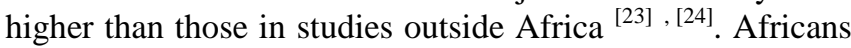
have been reported to have persistently elevated $\beta 2 \mathrm{M}$ levels as a result of recurrent or chronic malaria and helminthic infections. ${ }^{[23]}$ The higher baseline levels did not appear to impair the rise associated with HIV/TB or co infection with both diseases as there was there was still a statistically significant difference among the groups. This implies that immune activation continues to take place in those with HIV and or TB infection despite prior elevation. As an alternative to the CD4+ count however, the lack of correlation and the cost of 10USD at which each test was done makes it uneconomical now since it is more costly than the CD4+ count and adds no other benefit. The TST reaction in the study subjects was significantly lower than TB controls.

The tuberculin reaction size of $82 \%$ of study subjects who had CD4+ count less than 200cells/uL was $<5 \mathrm{~mm}$ (table 2). Mukadi et al also found negative TST reaction in those with low CD4 + level <200cells/ul. ${ }^{25}$ The finding is also in agreement with those of other. ${ }^{[24],[26]}$ The sensitivity of tuberculin skin testing is inversely related to the degree of immunosuppression. ${ }^{14}$ Depleted and dysfunctional CD4+ Tcells as found in HIV/TB co infection are unable to mount any immune reaction to antigens of invading organisms. ${ }^{[15],[}$ 26]

There was positive correlation between the TST reaction size and CD 4+ count indicating that those with higher TST reaction size had higher CD4+ count. However, predictive value of TST for CD4+ was weak at $31 \%$.

There was a statistically significant difference in the mean ESR of the study subjects compared to TB controls although both groups showed raised levels above normal reference values. This finding could be explained by the degree of inflammation and immune activation which is more marked in HIV/TB co-infection than in TB alone. ${ }^{7}$ The ESR showed negative correlation with CD4+count and also had a very weak $(8 \%)$ negative predictive value for $\mathrm{CD} 4+$ meaning that higher ESR is associated with lower CD4+count and vice-versa.

Conclusions: beta-2_microglobulin levels were higher in the study subjects than controls but there was no correlation with the CD4+ count. The ESR and TST had a weak and moderate predictive value for $\mathrm{CD} 4+$ count respectively.

Recommendations: The investigated immune activation markers i.e $\beta 2 \mathrm{M}$, TST and ESR were too weak to act as surrogates for CD4+. More research in needed in pursuit of a stronger and cheaper surrogates.

Study Limitations: Inability to measure other immnune activation markers like neopterin, tumour necrosis factor-alpha (TNF- $\alpha$ ) and gamma interferon due to cost and logistics. 


\section{ACKNOWLEDGEMENT}

Professor J.U OKPAPI, Department of Medicine, Ahmadu Bello University Teaching Hospital Zaria ,Nigeria

\section{REFERENCES}

[1] Gisseppe Pantaleo, Cecilia Graziosi, Anthony S Fauci. The immunopathogenesis of Human Immunodeficiency Virus Infection. N.Engl.J.Med. 1993; 328(5):327-334.

[2] Christof Geldmacher, Alexandra Schuetz, Njabulo Ngwenyama, Joseph P. Casazza, Erica Sanga, Elmar Saathoff et al: Early depletion of Mycobacterium tuberculosis-specific T helper 1 cell responses after HIV-1 infection: J Infect Dis. 2008 Dec 1; 198(11): 1590-1598. doi: $10.1086 / 593017$

[3] Fauci AS.The Human Immunodeficiency Virus: infectivity and mechanisms of pathogenesis. Science 1988; 239; 617-622.

[4] Jones BE, Young SMM, Antoriskis D. Relationship of tuberculosis Manifestations to CD4+ counts in patients with HIV infection. Am. Rev. Resp. Diseases 1993; 148:1291.52.

[5] Shafer RN, Edlin BR .Tuberculosis in patients infected with Human Immunodeficiency Virus: Perspectives on the last decade. Clin. Infect. Dis. 1996; 22:683-704.

[6] Vázquez EG, de Górgolas M, Guerrero ML. Relation between erythrocyte sedimentation rate, clinical and immune status and virus load in non-hospitalized HIV-infected patients. Rev Esp Quimioter. 2001;14:264-268

[7] M Hosp, IM Lisse, M. Quigley. An evaluation of low cost progression markers in HIV- 1 seropositive Zambians. (short communication).HIV Medicine.2000;1(2 ):125-127.

[8] Diane VH, Peter FB Tuberculosis in Patients with Human Immunodeficiency Virus Infection. N. Eng. J. Med. 1999; 340 (5):367-373.

[9] Nakata K, Rom WN, Honda Y et al. Mycobacterium tuberculosis enhances HIV-1 replication in the Lung. Am J. Resp. Crit Care Med. 1997; 155:996-1003.

[10] Wallis RS, Vjecha M, Amir-Tahmasseb $M$ et al. Influence of tuberculosis on human immunodeficiency virus (HIV-1) enhanced cytokine expression and elevated beta- 2- microglobulin in HIV-1associated tuberculosis. J. Infect. Dis. 1993; 167(1):43-48.

[11] Wanchu A, Arora S, Bhatnagar A, Sud A, Bambery P, Sing S. Decline in beta-2-Microglobulin Levels After Anti-tubercular Therapy in Tuberculosis Patients with HIV. The Indian Journal of Chest Diseases and Allied Sciences. 2001; 43(4):211--21517.

[12] Shi M, Taylor JM, Faley JL, Hoover DR, Munoz A. Kingsley LA. Early levels of CD4+, neopterin, and beta-2-microglobulins indicate future disease progression. J. Clin. Immunol 1997; 17(1):43-52.
[13] Wanchu A, Arora S, Bhagnathar A, Sud A, et al. Beta-2-microglobulin as surrogate marker for HIV infection: good correlation with CD4+ counts. Indian J.Pathol. Microbial.2004; 47(2):298-301.

[14] Gupta SM, Ray K, Bala M. Evaluation of beta-2-microglobulin level as a marker of HIV/AIDS progression. J. Commun. Dis.2004; 36 (3) :166-170.

[15] Sharma YV. Clinical utility of beta 2 microglobulin measurement. Med J Armed Forces India. 1997 Oct; 53 (4):249-250. doi:10.1016/S0377-1237(17)30745-1. Epub 2017 Jun 26

[16] S. D. Lawn, D. Rudolph, S. Wiktor*, D. Coulibaly*, A. Ackah* \& R. B Lal Tuberculosis (TB) and HIV infection are independently associated with elevated serum concentrations of tumour necrosis factor receptor type 1 and b2-microglobulin, respectively.Clin Exp Immunol 2000; $122: 79 \pm 84$

[17] Keiper MD, Beumont M, Eishami A, Langlotz CP, Miller WT Jr. CD4 ${ }^{+}$ $\mathrm{T}$ lymphocyte count and the radiographic presentation of pulmonary tuberculosis.A study of the relationship between these factors in patients with human immunodeficiency virus infection. Chest.1995; 107(1):74-80.

[18] R.Teck; O.Ascurra; P.Gomani et al. WHO clinical staging of HIV infection and disease, tuberculosis and eligibility for antiretroviral treatment: relationship to CD4 lymphocyte counts. The International Journal of Tuberculosis and Lung Disease. 2005; 9(3):258- 265.

[19] LawnSD, EvansAJ, SedwickPM, Achaempong JW. Pulmonary tuberculosis: radiological features in West Africans co-infected with HIV. The British Journal of Radiology, (1999); 72: 339-344.

[20] Kivihya-Ndugga LE, Ochola JJ, Otieno G, Muthami LN, Gathua S Clinical and immunological markers in Kenyan pulmonary tuberculosis patients with and without HIV-1. East Afr Med J, 1994;71 (6):373-375.

[21] Richter .C, Pallangyo KJ, Ndosi BN, Chum HJ, Swai AB, Shao J. Ches radiography and beta-2-microglobjulin levels in HIV-Seronegative and HIV-Seropositve African Patients with Pulmonary Tuberculosis. Trop. Geogr. Med. 1994; 46(5):283-287.

[22] Piwowar EM, Tugume SB, Grant RM et al. B-2 Microglobulin values among Human Immunodeficiency Virus (HIV)-Negative, HIV Positive asymptomatic, and HIV Positive Symptomatic Ugandans. Clinical and Diagnostic Laboratory Immunology.1995;2(2) P236-237.

[23] Pascale JM, Isaacs DM, Contreras P et al. Immunological Markers Of Disease Progression in Patients infected with The Human Immunodeficiency Virus. Clinical and Diagnostic Laboratory Immunology.1997; 4(4): 474-477.

[24] Mukadi Y, Perriens JH, St.Louis ME, et Al Spectrum Of Immunodeficiency In HIV-1- Infected Patients With Pulmonary Tuberculosis In Zaire. Lancet, 1993; 342 (8864): 143-146.

[25] Yusuph H, Garbati MA, Gashau W. Mantoux reaction in patients with HIV-related pulmonary tuberculosis in Maiduguri Nigeria Afr J Med Sci $2005 ; 34(2): 125-128$

[26] Ndakotsu MA, Salawu L, Durosinmi MA. Relation between erythrocyte sedimentation rate, clinical and immune status in HIV-infected patients. Niger J Med. 2008;17: 420-422. 